\title{
AN ANALYTICAL SOLUTION FOR THREE-DIMENSIONAL DIFFRACTION OF PLANE P-WAVES BY A HEMISPHERICAL ALLUVIAL VALLEY WITH SATURATED SOIL DEPOSITS ${ }^{\star \star}$
}

\author{
Zhao Chenggang Dong Jun \\ (School of Civil Engineering and Architecture, Beijing Jiaotong University, Beijing 100044, China) \\ Gao Fuping ${ }^{\star} 1$ \\ (Institute of Mechanics, Chinese Academy of Sciences, Beijing 100080, China)
}

Received 13 January 2005; revision received 30 April 2006

\begin{abstract}
An analytical solution for the three-dimensional scattering and diffraction of plane $\mathrm{P}$-waves by a hemispherical alluvial valley with saturated soil deposits is developed by employing Fourier-Bessel series expansion technique. Unlike previous studies, in which the saturated soil deposits were simulated with the single-phase elastic theory, in this paper, they are simulated with Biot's dynamic theory for saturated porous media, and the half space is assumed as a single-phase elastic medium. The effects of the dimensionless frequency, the incidence angle of $\mathrm{P}$-wave and the porosity of soil deposits on the surface displacement magnifications of the hemispherical alluvial valley are investigated. Numerical results show that the existence of a saturated hemispherical alluvial valley has much influence on the surface displacement magnifications. It is more reasonable to simulate soil deposits with Biot's dynamic theory when evaluating the displacement responses of a hemispherical alluvial valley with an incidence of $\mathrm{P}$-waves.
\end{abstract}

KEY WORDS Biot's dynamic theory, three-dimensional scattering, hemispherical alluvial valley, analytical solution

\section{INTRODUCTION}

Irregular local geological conditions and topography may have significant influence on the amplification of the ground motion and thereby cause earthquake disasters, especially for the situations where thick soft clay deposit exists. A typical example is the Mexico earthquake in $1985^{[1]}$. The earthquake occurred on the coast of Michoacan, USA (magnitude is 8.1), however Mexico City was damaged even more seriously than the epicenter. Investigations showed that it is the thick bathtub soft clay stratification on which Mexico City laiy that caused these results. Therefore, to investigate the dynamic response of a field with special local geological conditions is of great importance for earthquake resistance design.

In the recent three decades, the effects of irregular geological conditions and surface topography on the amplification of the ground motion have been drawing increasing attention from geotechnical

\footnotetext{
* Corresponding author. Tel:+86-10-62618499, Fax:+86-10-62561284, Email:fpgao@inech.ac.cn

$\star \star$ Project supported by the National Natural Science Foundation of China (No. 50478062) and Natural Science Foundation of Beijing (No. 8052015).
} 
engineers, seismologists and earthquake engineers. Since Trifunac ${ }^{[2]}$ developed for the first time an analytical solution for scattering of plane $\mathrm{SH}$-waves by a semicircular canyon, great progress has been made on this subject ${ }^{[2--6]}$. Some researchers further investigated the three-dimensional cases ${ }^{[7--9]}$. Lee $^{[8,9]}$ developed an analytical solution for three-dimensional scattering and diffraction of plane waves by hemispherical canyons and hemispherical alluvial valleys. However, in the above analytical investigations the soft clay deposit in a canyon or alluvial valley was regarded as single-phase elastic medium. It would be more reasonable to simulate the soft clay deposit with saturated porous media. So far, only a few two-dimensional models have been developed for analysis of the scattering and diffraction of plane waves in a saturated porous medium by irregular geological conditions ${ }^{[10,11]}$.

In this paper, an analytical solution is developed for the three-dimensional scattering and diffraction of $\mathrm{P}$-wave by a saturated hemispherical alluvial valley, where the soft deposit is simulated by Biot's dynamic theory ${ }^{[12,16]}$ and the half space is assumed as single-phase elastic media. The surface displacement amplitude of a hemispherical alluvial valley is obtained analytically. The effects of the frequency and angle of incidence of $\mathrm{P}$-wave and the porosity of sediments on the surface displacement amplitudes are discussed. Furthermore, these results are compared with those obtained by Lee ${ }^{[9]}$, in which the alluvial valley was assumed as a single-phase elastic medium.

\section{THE MODEL AND FUNDAMENTAL EQUATIONS}

Consider a three-dimensional soft saturated porous hemispherical alluvial valley, with radius $r=a$, embedded in a half-space, as shown in Fig.1. The half-space is assumed as a homogeneous and isotropic single-phase elastic medium, and the hemispherical alluvial valley as a homogeneous saturated porous medium. The interface between the two media is assumed to be impervious. The geometry of the model is described with spherical coordinates $(r, \theta, \varphi)$ (see Fig.2). For the study of surface displacement, rectangular coordinates are also employed. The relationship between the two coordinates is shown in Fig.2.

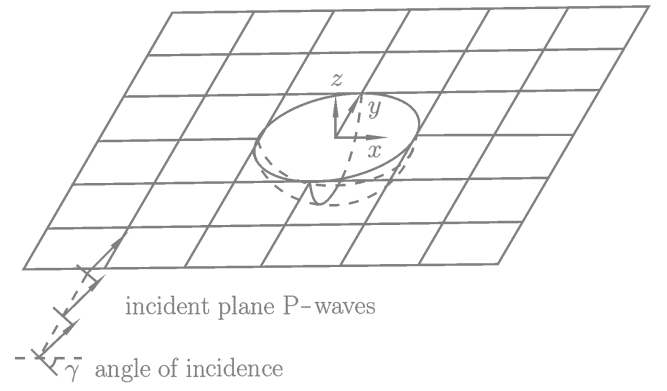

Fig. 1. An illustrationof a half-space with a hemisphere alluvial valley and incident $\mathrm{P}$-waves.

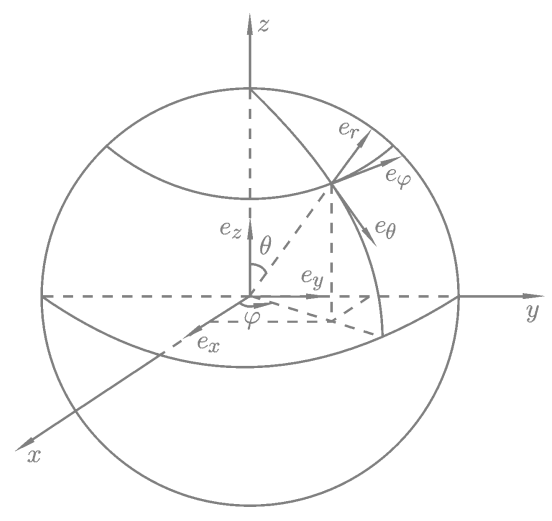

Fig. 2. Spherical and rectangular coordinates.

The fundamental equations for the soft saturated porous hemispherical alluvial valley are chosen as Biot's dynamic equations ${ }^{[12]}$. Meanwhile, the traditional single-phase elastic theory is employed for simulating the half space.

By defining $F(r, \theta, \varphi, t)$ as a function of space and time, the wave equation in spherical coordinates can be written as

$$
\frac{1}{r^{2}} \frac{\partial}{\partial r}\left(r^{2} \frac{\partial F}{\partial r}\right)+\frac{1}{r^{2} \sin \theta} \frac{\partial}{\partial \theta}\left(\sin \theta \frac{\partial F}{\partial \theta}\right)+\frac{1}{r^{2} \sin ^{2} \theta} \frac{\partial^{2} F}{\partial \varphi^{2}}-\frac{1}{c^{2}} \frac{\partial^{2} F}{\partial t^{2}}=0
$$

By means of separation of variables, a general harmonic solution of Eq.(1) is a linear combination of any two of the four basic solutions of the form

$$
Z_{n}^{j}(k r) P_{n}^{m}(\mu)_{\sin }^{\cos }(m \varphi) \exp (-\mathrm{i} \omega t)
$$


where $Z_{n}^{j}(j=1,2,3,4)$ are the spherical Bessel and Hankel functions:

$$
\begin{array}{ll}
Z_{n}^{(1)}(k r)=j_{n}(k r)=\left(\frac{\pi}{2 k r}\right)^{1 / 2} J_{n+1 / 2}(k r), & Z_{n}^{(2)}(k r)=y_{n}(k r)=\left(\frac{\pi}{2 k r}\right)^{1 / 2} Y_{n+1 / 2}(k r) \\
Z_{n}^{(3)}(k r)=h_{n}^{(1)}(k r)=\left(\frac{\pi}{2 k r}\right)^{1 / 2} H_{n+1 / 2}^{(1)}(k r), & Z_{n}^{(4)}(k r)=h_{n}^{(2)}(k r)=\left(\frac{\pi}{2 k r}\right)^{1 / 2} H_{n+1 / 2}^{(2)}(k r)
\end{array}
$$

in which $j_{n}(k r), y_{n}(k r)$ and $h_{n}(k r)$ are the cylindrical Bessel and Hankel functions, $k$ is the wave number, $\mathrm{i}=\sqrt{-1}, m \leq n=0,1,2, \ldots, P_{n}^{m}(\mu)$ is the associated Legendre polynomial, and $\mu=\cos \theta$.

\section{SOLUTION OF THE PROBLEM}

In this study, we consider P-waves are propagating from the elastic half-space to a hemispherical alluvial valley with an angle of incidence $\gamma$ relative to $z$-axis, as shown in Fig.1. In solving Biot's governing equations, it is convenient to introduce the following Helmholtz resolution ${ }^{[12]}$ :

$$
\boldsymbol{u}=\operatorname{grad} \Phi+\operatorname{curl} \boldsymbol{H}, \quad \boldsymbol{U}=\operatorname{grad} \Psi+\operatorname{curl} \boldsymbol{G}
$$

where $\Phi$ and $\boldsymbol{H}$ are potentials associated with the solid phase of the saturated porous material, while $\Psi$ and $\boldsymbol{G}$ are potentials associated with the pore fluid phase.

The displacement vector of incident plane P-waves in the elastic half-space can be expressed as

$$
u^{i}=a_{0}\left(\sin \gamma e_{x}+\cos \gamma e_{z}\right) \exp \left[\mathrm{i} h_{0}(x \sin \gamma+z \cos \gamma)-\mathrm{i} \omega t\right]
$$

in which $a_{0}$ is the amplitude of incident P-waves, $\omega$ the angular frequency. In general, for the presence of only free half-space boundary, the incident P-wave is reflected from the plane free surface $(z=0)$ into $\mathrm{P}$-wave and SV-wave, whose displacement vectors can be given as follows, respectively

$$
\begin{aligned}
& u_{1}^{r}=K_{1} a_{0}\left(\sin \gamma e_{x}+\cos \gamma e_{z}\right) \exp \left[\mathrm{i} h_{0}(x \sin \gamma-z \cos \gamma)\right] \\
& u_{2}^{r}=K_{2}\left(\alpha_{0} / \beta_{0}\right) a_{0}\left(\cos \delta e_{x}+\sin \delta e_{z}\right) \times \exp \left[\mathrm{i} k_{0}(x \sin \delta-z \cos \delta)\right]
\end{aligned}
$$

where $e_{x}$ and $e_{z}$ are the unit vectors in $x$ - and $z$-directions, $\alpha_{0}$ and $\beta_{0}$ are the P-wave velocity and $\mathrm{SV}$-wave velocity, respectively, $h_{0}=\omega / \alpha_{0}$ is the wave number of $\mathrm{P}$-wave, $k_{0}=\omega / \beta_{0}$ is the wave number of SV-wave, superscript ' $r$ ' means the reflected waves, the subscripts ' 1 ' and ' 2 ' represent P-wave and $\mathrm{SV}$-wave, respectively. $K_{1}$ and $K_{2}$ are the reflection coefficients, which are given as

$$
\begin{aligned}
& \sin \delta=\frac{\beta_{0}}{\alpha_{0}} \sin \gamma \\
& K_{1}=\frac{\sin (2 \gamma) \sin (2 \delta)-\left(\alpha_{0} / \beta_{0}\right)^{2} \cos ^{2}(2 \delta)}{\sin (2 \gamma) \sin (2 \delta)+\left(\alpha_{0} / \beta_{0}\right)^{2} \cos ^{2}(2 \delta)} \\
& K_{2}=\frac{2 \sin (2 \gamma) \cos (2 \delta)}{\sin (2 \gamma) \sin (2 \delta)+\left(\alpha_{0} / \beta_{0}\right)^{2} \cos ^{2}(2 \delta)}
\end{aligned}
$$

The resultant displacement vector is

$$
u^{i+r}=u^{i}+u_{1}^{r}+u_{2}^{r}
$$

The stress-free boundary conditions should be satisfied, i.e.

$$
\sigma_{z z}^{i+r}=\sigma_{z x}^{i+r}=\sigma_{z y}^{i+r}=0 \quad \text { at } \quad z=0
$$

Based on the theory for elastic waves, it is convenient to employ wave potentials to get the displacement solutions of $u$. Thus $u^{i}$ and $u_{1}^{r}$ can be derived from $\phi^{(i)}, \phi^{(r)}$, respectively, and $u_{2}^{r}$ from $\psi^{(r)}$ and $\chi^{(r)}$. These wave potentials can be expanded as

$$
\begin{aligned}
\phi^{(i)} & =\sum_{m, n} A_{m n}^{(i)} j_{n}\left(h_{0} r\right) P_{n}^{m}(\mu) \cos (m \varphi) \\
\psi^{(i)} & =\chi^{(i)}=B_{m n}^{(i)}=C_{m n}^{(i)}=0 \\
\phi^{(r)} & =\sum_{m, n} A_{m n}^{(r)} j_{n}\left(h_{0} r\right) P_{n}^{m}(\mu) \cos (m \varphi) \\
\psi^{(r)} & =\sum_{m, n}^{m} k_{0} B_{m n}^{(r)} j_{n}\left(k_{0} r\right) P_{n}^{m}(\mu) \sin (m \varphi) \\
\chi^{(r)} & =\sum_{m, n}^{m} C_{m n}^{(r)} j_{n}\left(k_{0} r\right) P_{n}^{m}(\mu) \cos (m \varphi)
\end{aligned}
$$


where the summation is for $m \leq n=0,1,2, \ldots$, and the coefficients $\left\{A_{m n}^{(i)}, A_{m n}^{(r)}, B_{m n}^{(r)}, C_{m n}^{(r)}\right\}$ were given in Ref.[8]. The above potentials should satisfy their scalar wave equations:

$$
\nabla^{2}\left\{\begin{array}{l}
\phi^{(j)} \\
\psi^{(j)} \\
\chi^{(j)}
\end{array}\right\}+\left\{\begin{array}{c}
h_{j}^{2} \phi^{(j)} \\
k_{j}^{2} \psi^{(j)} \\
k_{j}^{2} \chi^{(j)}
\end{array}\right\}=0
$$

where $h_{j}$ and $k_{j}$ are the wave number of $\mathrm{P}$-wave and that of SV-wave in the elastic half-space, respectively.

For the presence of the saturated alluvial valley, both longitudinal and transverse outgoing spherical waves are reflected back into the valley, where standing waves are formed. The potentials of reflected waves by the spherical boundary surface can be expressed as

$$
\begin{aligned}
\phi^{(S)} & =\sum_{m, n} A_{m n}^{(3)} Z_{n}^{(3)}\left(h_{0} r\right) P_{n}^{m}(\mu) \cos (m \varphi) \\
\psi^{(S)} & =\sum_{m, n} k_{0} B_{m n}^{(3)} Z_{n}^{(3)}\left(k_{0} r\right) P_{n}^{m}(\mu) \sin (m \varphi) \\
\chi^{(S)} & =\sum_{m, n}^{m,} C_{m n}^{(3)} Z_{n}^{(3)}\left(k_{0} r\right) P_{n}^{m}(\mu) \cos (m \varphi)
\end{aligned}
$$

where the summation is for $m \leq n=0,1,2, \ldots$, the spherical Hankel function $Z_{n}^{(3)}=h_{n}^{(1)}$ is used for the product $h_{n}^{(1)}(\cdot) \exp (-\mathrm{i} \omega t)$, which represents an outward propagating-wave, the superscript ' $S$ ' means reflection by the spherical boundary surface in half-space.

According to Biot's dynamic theory ${ }^{[12]}$, longitudinal $\mathrm{P}_{\mathrm{I}}$-wave, $\mathrm{P}_{\mathrm{II}}$-wave and transverse $\mathrm{SV}$-wave will be refracted in the valley. The refracted standing wave potentials can be represented by ${ }^{[15]}$

$$
\begin{array}{rlrl}
\phi_{1}^{(f)} & =\sum_{m, n} A_{m n}^{(f)} j_{n}^{(1)}\left(h_{1} r\right) P_{n}^{m}(\mu) \cos (m \varphi), & \phi_{2}^{(f)} & =\sum_{m, n} B_{m n}^{(f)} j_{n}^{(1)}\left(h_{2} r\right) P_{n}^{m}(\mu) \cos (m \varphi) \\
\psi^{(f)} & =\sum_{m, n} k_{1} C_{m n}^{(f)} j_{n}^{(3)}\left(k_{1} r\right) P_{n}^{m}(\mu) \sin (m \varphi), & \chi^{(f)}=\sum_{m, n}^{\infty} D_{m n}^{(f)} j_{n}^{(1)}\left(k_{1} r\right) P_{n}^{m}(\mu) \cos (m \varphi)
\end{array}
$$

where superscript ' $f$ ' means refraction by the spherical boundary surface, $h_{1}\left(=\omega / \alpha_{1}\right)$ and $h_{2}\left(=\omega / \alpha_{2}\right)$ are the wave numbers of $\mathrm{P}_{\mathrm{I}}$ and $\mathrm{P}_{\mathrm{II}}$-waves, respectively, $k_{1}\left(=\omega / \beta_{1}\right)$ is the wave number of transverse wave in the saturated alluvial valley, $\left\{A_{m n}^{(3)}, B_{m n}^{(3)}, C_{m n}^{(3)}\right\}$ and $\left\{A_{m n}^{(f)}, B_{m n}^{(f)}, C_{m n}^{(f)}, D_{m n}^{(f)}\right\}$ are sets of expansion coefficients corresponding to scattered and refracted potentials $\left\{\phi^{(S)}, \psi^{(S)}, \chi^{(S)}\right\}$ and $\left\{\phi_{1}^{(f)}, \phi_{2}^{(f)}, \psi^{(f)}\right.$, $\left.\chi^{(f)}\right\}$, which satisfy the associated scalar wave equations (11). The displacements can thereby be expressed by the spherical coordinate as

$$
\begin{aligned}
& u_{r}=\frac{\partial \phi}{\partial r}+\left[\frac{\partial^{2}(r \chi)}{\partial r^{2}}-r \nabla^{2} \chi\right] \\
& u_{\theta}=\frac{1}{r} \frac{\partial \phi}{\partial \theta}+\frac{1}{r \sin \theta} \frac{\partial(r \psi)}{\partial \varphi}+\frac{1}{r} \frac{\partial^{2}(r \chi)}{\partial \theta \partial r} \\
& u_{\varphi}=\frac{1}{r \sin \theta} \frac{\partial \phi}{\partial \varphi}-\frac{1}{r} \frac{\partial(r \psi)}{\partial \theta}+\frac{1}{r \sin \theta} \frac{\partial^{2}(r \chi)}{\partial \varphi \partial r}
\end{aligned}
$$

By satisfying the boundary conditions, the expansion coefficients can be determined. The boundary conditions in the spherical boundary surface $(r=a)$ are

$$
u^{i}+u^{r}+u^{s}=u^{f}, \quad \sigma^{i}+\sigma^{r}+\sigma^{s}=\sigma_{s}^{f}+\sigma_{l}^{f}
$$

and the impervious boundary condition is

$$
u_{s, r}^{f}-u_{l, r}^{f}=0
$$


In the presence of free boundary $(z=0)$ and the hemispherical valley $(r \leq a, z \leq 0)$, additional spherical wave potentials will be generated in both the half-space medium and the saturated alluvial valley. They can be presented as follows:

(i) Half-space:

$$
\begin{aligned}
\phi^{(R)} & =\sum_{j, m, n} A_{m n}^{(j)} Z_{n}^{(j)}\left(h_{0} r\right) P_{n}^{m}(\mu) \cos (m \varphi) \\
\psi^{(R)} & =\sum_{j, m, n}^{j} k_{0} B_{m n}^{(j)} Z_{n}^{(j)}\left(k_{0} r\right) P_{n}^{m}(\mu) \sin (m \varphi) \\
\chi^{(R)} & =\sum_{j, m, n} C_{m n}^{(j)} Z_{n}^{(j)}\left(k_{0} r\right) P_{n}^{m}(\mu) \cos (m \varphi)
\end{aligned}
$$

where $j=1,2, m \leq n=0,1,2, \ldots$, the subscript ' $R$ ' means the scattering between the free boundary surface and the spherical boundary surface in half-space.

(ii) Saturated alluvial valley:

$$
\begin{array}{lll}
\phi_{1}^{(F)}=\sum_{m, n} A_{m n}^{(F)} j_{n}^{(1)}\left(h_{1} r\right) P_{n}^{m}(\mu) \cos (m \varphi), & \phi_{2}^{(F)}=\sum_{m, n} B_{m n}^{(F)} j_{n}^{(1)}\left(h_{2} r\right) P_{n}^{m}(\mu) \cos (m \varphi) \\
\psi^{(F)}=\sum_{j, m, n} k_{1} C_{m n}^{(F)} j_{n}^{(1)}\left(k_{1} r\right) P_{n}^{m}(\mu) \sin (m \varphi), & \chi^{(F)}=\sum_{m, n} D_{m n}^{(F)} j_{n}^{(1)}\left(k_{1} r\right) P_{n}^{m}(\mu) \cos (m \varphi)
\end{array}
$$

where $m \leq n=0,1,2, \ldots$; the subscript ' $F$ ' means the reflection by the free boundary surface in the saturated porous material. The potentials $\left\{\phi^{(R)}, \psi^{(R)}, \chi^{(R)}\right\}$ and $\left\{\phi_{1}^{(F)}, \phi_{1}^{(F)}, \psi^{(F)}, \chi^{(F)}\right\}$ satisfy the associated scalar wave equations (11). The unknown coefficients $\left\{A_{m n}^{(1)}, B_{m n}^{(1)}, C_{m n}^{(1)}\right\},\left\{A_{m n}^{(2)}, B_{m n}^{(2)}, C_{m n}^{(2)}\right\}$ and $\left\{A_{m n}^{(F)}, B_{m n}^{(F)}, C_{m n}^{(F)}, D_{m n}^{(F)}\right\}$ can be determined with given boundary conditions.

The resultant potentials $\phi$ for the whole wave field are given by the sum of the potentials in each medium. Similarly, the resultant displacements $\boldsymbol{u}$ and the stress vector $\boldsymbol{\sigma}$ can be obtained. They are expressed as follows:

(i) Half-space (represented by superscript ' 0 '):

Potentials:

$$
\begin{aligned}
& \phi^{(0)}=\phi^{(i)}+\phi^{(r)}+\phi^{(S)}+\phi^{(R)} \\
& \psi^{(0)}=\psi^{(i)}+\psi^{(r)}+\psi^{(S)}+\psi^{(R)} \\
& \chi^{(0)}=\chi^{(i)}+\chi^{(r)}+\chi^{(S)}+\chi^{(R)}
\end{aligned}
$$

Displacements:

$$
u^{(0)}=u^{i}+u^{r}+u^{S}+u^{R}
$$

Stresses:

$$
\sigma^{(0)}=\sigma^{i}+\sigma^{r}+\sigma^{S}+\sigma^{R}
$$

(ii) Saturated alluvial valley (represented by superscript ' 1 '):

(1) Solid phase:

Scalar wave potentials :

$$
\phi_{1}^{(1)}=\phi_{1}^{(f)}+\phi_{1}^{(F)}, \quad \phi_{2}^{(1)}=\phi_{2}^{(f)}+\phi_{2}^{(F)}
$$

Vector wave potentials $\boldsymbol{H}$ :

$$
\psi^{(1)}=\psi^{(f)}+\psi^{(F)}, \quad \chi^{(1)}=\chi^{(f)}+\chi^{(F)}
$$

Displacements:

$$
u_{s}^{(1)}=u_{s}^{f}+u_{s}^{F}
$$

Stresses:

$$
\sigma_{s}^{(1)}=\sigma_{s}^{f}+\sigma_{s}^{F}
$$

(2) Liquid phase:

Scalar wave potentials $\Psi^{\prime}$ : 


$$
\phi_{1}^{\prime(1)}=\eta_{\mathrm{I}}\left(\phi_{1}^{(f)}+\phi_{1}^{(F)}\right), \quad \phi_{2}^{\prime(1)}=\eta_{\mathrm{II}}\left(\phi_{2}^{(f)}+\phi_{2}^{(F)}\right)
$$

Vector wave potentials $\boldsymbol{G}$ :

$$
\psi^{\prime(1)}=\eta_{\mathrm{III}}\left(\psi^{(f)}+\psi^{(F)}\right), \quad \chi^{\prime(1)}=\eta_{\mathrm{III}}\left(\chi^{(f)}+\chi^{(F)}\right)
$$

Displacements:

$$
u_{l}^{(1)}=u_{l}^{f}+u_{l}^{F}
$$

Stresses:

$$
\sigma_{l}^{(1)}=\sigma_{l}^{f}+\sigma_{l}^{F}
$$

where $\eta_{\mathrm{I}}, \eta_{\mathrm{II}}, \eta_{\mathrm{III}}$ are the amplitude ratios of potentials for the solid and fluid phases of the saturated porous media $^{[13]}$.

The following boundary conditions should be satisfied:

(a) Valley and half-space interface conditions:

The displacements and stresses along the interface between the valley and the half-space $(r=a)$ are assumed identical:

$$
\begin{aligned}
& u_{s}^{(0)}=u^{i}+u^{r}+u^{s}+u^{R}=u_{s}^{f}+u_{s}^{F}=u_{s}^{(1)} \\
& \sigma^{(0)}=\sigma^{i}+\sigma^{r}+\sigma^{s}+\sigma^{R}=\sigma_{s}^{f}+\sigma_{s}^{F}+\sigma_{l}^{f+F}=\sigma^{(1)}
\end{aligned}
$$

The interface is assumed to be impervious, i.e.

$$
u_{s, r}^{(1)} f+F-u_{l, r}^{(1)} f+F=0
$$

Combining Eqs.(15),(16), (30) and (31) gives (at $r=a, z<0)$

$$
u^{R}=u_{s}^{F}, \quad \sigma^{R}=\sigma_{s}^{F}+\sigma_{l}^{F}, \quad u_{s, r}^{F}-u_{l, r}^{F}=0
$$

(b) Free surface conditions:

(1) Half-space:

Along the free surface of the half-space $\left(z=0, x^{2}+y^{2} \geq a^{2}\right)$, the wave-induced stresses vanish, i.e.

$$
\sigma_{z z}^{(0)}=\sigma_{z x}^{(0)}=\sigma_{z y}^{(0)}=0
$$

In the spherical coordinate, this equation can be transformed into

$$
\sigma_{\theta \theta}^{(0)}=\sigma_{\theta r}^{(0)}=\sigma_{\theta \varphi}^{(0)}=0 \quad \text { at } \quad r \geq a, \quad 0 \leq \varphi \leq 2 \pi, \quad \theta=\pi / 2
$$

Combining boundary condition Eqs.(9) and (34), we get

$$
\sigma^{S}+\sigma^{R}=0 \quad \text { at } \quad r>a, \quad z=0
$$

(2) Saturated alluvial valley:

At the free surface of the saturated alluvial valley $\left(z=0, x^{2}+y^{2}<a^{2}\right)$, the wave-induced stresses vanish, i.e.

$$
\sigma_{z z}^{(1)}=\sigma_{z x}^{(1)}=\sigma_{z y}^{(1)}=0
$$

In the spherical coordinate, Eq.(36) can be transformed into

$$
\sigma_{\theta \theta}^{(1)}=\sigma_{\theta r}^{(1)}=\sigma_{\theta \varphi}^{(1)}=0 \quad \text { at } \quad r<a, \quad 0 \leq \varphi \leq 2 \pi, \quad \theta=\pi / 2
$$

Assuming the free surface of the valley is permeable for water, the permeable boundary conditions are

$$
\sigma_{l, r r}^{(1)}=0 \quad \text { at } \quad r<a, \quad z=0
$$


Similar to the analysis by Lee ${ }^{[14]}$, using Eqs.(30), (31), (4) and (5) for each ' $(m, n)$ component' and $m \leq n=0,1,2, \ldots$, the following formulae can be obtained (at $r=a$ ):

$$
\begin{gathered}
\sum_{j} A_{m n}^{(j)} d_{0,11}^{(j)}+C_{m n}^{(j)} d_{0,13}^{(j)}=A_{m n}^{(F)} d_{1,11}^{(1)}+B_{m n}^{(F)} d_{1,12}^{(1)}+D_{m n}^{(F)} d_{1,14}^{(1)} \\
\sum_{j} A_{m n}^{(j)} d_{0,21}^{(j)}+C_{m n}^{(j)} d_{0,23}^{(j)}=A_{m n}^{(F)} d_{1,21}^{(1)}+B_{m n}^{(F)} d_{1,22}^{(1)}+D_{m n}^{(F)} d_{1,24}^{(1)} \\
\sum_{j} k_{0} B_{m n}^{(j)} d_{0,22}^{(j)}=k_{1} C_{m n}^{(F)} d_{1,23}^{(1)} \\
\sum_{j} A_{m n}^{(j)} E_{0,11}^{(j)}+C_{m n}^{(j)} E_{0,13}^{(j)}= \\
\sum_{j} A_{m n}^{(j)} E_{0,41}^{(j)}+C_{m n}^{(j)} E_{0,43}^{(j)}=A_{m n}^{(F)} E_{1,41}^{(1)}+B_{m n}^{(F)} E_{1,42}^{(1)}+D_{m n}^{(F)} E_{1,44}^{(1)} E_{1,11}^{(1)}+\left(1+\eta_{2}\right) B_{m n}^{(F)} E_{1,12}^{(1)}+\left(1+\eta_{3}\right) D_{m n}^{(F)} E_{1,14}^{(1)} \\
\sum_{j} B_{m n}^{(j)} E_{0,42}^{(j)}=C_{m n}^{(F)} E_{1,43}^{(1)} \\
A_{m n}^{(F)} d_{1,11}^{(1)}+B_{m n}^{(F)} d_{1,12}^{(1)}+D_{m n}^{(F)} d_{1,14}^{(1)}=\eta_{1} A_{m n}^{(F)} d_{1,11}^{(1)}+\eta_{2} B_{m n}^{(F)} d_{1,12}^{(1)}+\eta_{3} D_{m n}^{(F)} d_{1,14}^{(1)}
\end{gathered}
$$

with $j=1,2$ in the summation. The terms $d_{0, i j}^{(k)}, E_{0, i j}^{(k)}, d_{1, i j}^{(k)}, E_{1, i j}^{(k)}$ are coefficients of the equations. $d_{0, i j}^{(k)}, E_{0, i j}^{(k)}$ are given by Lee ${ }^{[14]}, d_{1, i j}^{(k)}, E_{1, i j}^{(k)}$ can be calculated with the saturated porous media in the same way. Equations (39) have ten unknown coefficients $\left\{A_{m n}^{(1)}, B_{m n}^{(1)}, C_{m n}^{(1)}\right\},\left\{A_{m n}^{(2)}, B_{m n}^{(2)}, C_{m n}^{(2)}\right\}$, $\left\{A_{m n}^{(F)}, B_{m n}^{(F)}, C_{m n}^{(F)}, D_{m n}^{(F)}\right\}$. In this analysis, the boundary conditions, i.e. Eqs.(33) (38), are used. On the free surface of half-space $(z=0)$, the spherical wave potentials are represented by series with argument ' $r$ ', and putting these into Eqs.(33) (35), at $\theta=\pi / 2,0 \leq \varphi \leq 2 \pi, r \geq \mathrm{a}, \sigma_{\theta \theta}=0$, for $m=0,1,2, \ldots$, we get

$$
\begin{aligned}
& \sum_{l=0}^{n}\left(\begin{array}{c}
A_{m, m+2 l}^{(1)} \\
A_{m, m+2 l}^{(3)}
\end{array}\right) e_{0,21}^{(1)}+\left(+\begin{array}{c}
B_{m, m+2 l-1}^{(1)} \\
B_{m, m+2 l-1}^{(3)}
\end{array}\right) e_{0,22}^{(1)}+\left(\begin{array}{c}
C_{m, m+2 l}^{(1)} \\
C_{m, m+2 l}^{(3)}
\end{array}\right) e_{0,23}^{(1)}=0 \quad(n=0,1,2, \ldots) \\
& \sum_{l=\max (0, n)}^{\infty}\left(+\begin{array}{c}
A_{m, m+2 l}^{(2)} \\
\mathrm{i} A_{m, m+2 l}^{(3)}
\end{array}\right) e_{0,21}^{(2)}+\left(+\begin{array}{c}
B_{m, m+2 l+1}^{(2)} \\
\mathrm{i} B_{m, m+2 l+1}^{(3)}
\end{array}\right) e_{0,22}^{(2)}+\left(+{ }_{\mathrm{i} C_{m, m+2 l}^{(3)}}^{C_{m, m+2 l}^{(2)}}\right) e_{0,23}^{(2)}=0 \quad(n=0, \pm 1, \pm 2, \ldots)
\end{aligned}
$$

Similar to $\sigma_{\theta r}=0, m=0,1,2, \ldots$, we get

$$
\begin{aligned}
& \sum_{l=0}^{n}\left(+\begin{array}{c}
A_{m, m+2 l+1}^{(1)} \\
A_{m, m+2 l+1}^{(3)}
\end{array}\right) e_{0,41}^{(1)}+\left(+\begin{array}{c}
B_{m, m+2 l}^{(1)} \\
B_{m, m+2 l}^{(3)}
\end{array}\right) e_{0,42}^{(1)}+\left(+\begin{array}{c}
C_{m, m+2 l+1}^{(1)} \\
C_{m, m+2 l+1}^{(3)}
\end{array}\right) e_{0,43}^{(1)}=0 \quad(n=0,1,2, \ldots) \\
& \sum_{l=\max (0, n)}^{\infty}\left(\begin{array}{c}
A_{m, m+2 l-1}^{(2)} \\
\mathrm{i} A_{m, m+2 l-1}^{(3)}
\end{array}\right) e_{0,41}^{(2)}+\left(\begin{array}{c}
B_{m, m+2 l}^{(2)} \\
\mathrm{i} B_{m, m+2 l}^{(3)}
\end{array}\right) e_{0,42}^{(2)}+\left(\begin{array}{c}
C_{m, m+2 l-1}^{(2)} \\
{ }_{\mathrm{i} C_{m, m+2 l-1}^{(3)}}^{(3)}
\end{array}\right) e_{0,43}^{(2)}=0 \quad(n=0, \pm 1, \pm 2, \ldots)
\end{aligned}
$$

For $\sigma_{\theta \varphi}=0, m=0,1,2, \ldots$, we get

$$
\begin{aligned}
& \sum_{l=0}^{n}\left(+\begin{array}{c}
A_{m, m+2 l+1}^{(1)} \\
A_{m, m+2 l+1}^{(3)}
\end{array}\right) e_{0,61}^{(1)}+\left(+\begin{array}{c}
B_{m, m+2 l}^{(1)} \\
B_{m, m+2 l}^{(3)}
\end{array}\right) e_{0,62}^{(1)}+\left(\begin{array}{c}
C_{m, m+2 l+1}^{(1)} \\
C_{m, m+2 l+1}^{(3)}
\end{array}\right) e_{0,63}^{(1)}=0 \quad(n=0,1,2, \ldots) \\
& \sum_{l=\max (0, n)}^{\infty}\left(+\begin{array}{c}
A_{m, m+2 l-1}^{(2)} \\
\mathrm{i} A_{m, m+2 l-1}^{(3)}
\end{array}\right) e_{0,61}^{(2)}+\left(\begin{array}{c}
B_{m, m+2 l}^{(2)} \\
{ }_{\mathrm{i} B_{m, m+2 l}^{(3)}}^{(3)}
\end{array}\right) e_{0,62}^{(2)}+\left(\begin{array}{c}
C_{m, m+2 l-1}^{(2)} \\
{ }_{\mathrm{i} C_{m, m+2 l-1}^{(3)}}^{(2)}
\end{array}\right) e_{0,63}^{(2)}=0 \quad(n=0, \pm 1, \pm 2, \ldots)
\end{aligned}
$$

where $\mathrm{i}=\sqrt{-1}, e_{0, i j}^{(k)}$ are series expansion coefficients given by Lee ${ }^{[14]}$. Similar to the above free surface of the saturated alluvial valley, Eqs.(36) (38) are used, at $\theta=\pi / 2,0 \leq \varphi \leq 2 \pi, r \leq a, \sigma_{\theta \theta}=0$, for $m=0,1,2, \ldots$, we get

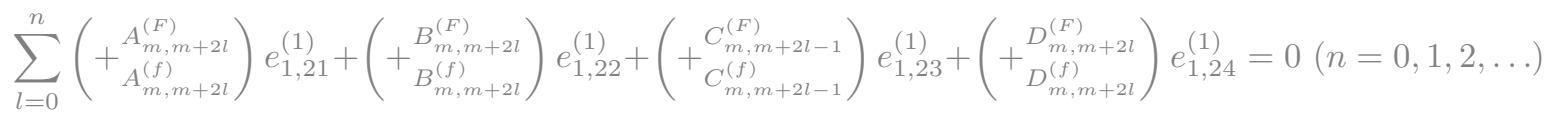


Similarly for $\sigma_{\theta r}=0, m=0,1,2, \ldots$, we get

$$
\begin{aligned}
& \sum_{l=0}^{n}\left(\begin{array}{c}
A_{m, m+2 l+1}^{(F)} \\
A_{m, m+2 l+1}^{(f)}
\end{array}\right) e_{1,41}^{(1)}+\left(+\begin{array}{c}
B_{m, m+2 l+1}^{(F)} \\
B_{m, m+2 l+1}^{(f)}
\end{array}\right) e_{1,42}^{(1)} \\
& \quad+\left(\begin{array}{c}
C_{m, m+2 l}^{(F)} \\
C_{m, m+2 l}^{(f)}
\end{array}\right) e_{1,43}^{(1)}+\left(+\begin{array}{c}
D_{m, m+2 l+1}^{(F)} \\
D_{m, m+2 l+1}^{(f)}
\end{array}\right) e_{1,44}^{(1)}=0 \quad(n=0,1,2, \ldots)
\end{aligned}
$$

For $\sigma_{\theta \varphi}=0, m=0,1,2, \ldots$, we get

$$
\begin{aligned}
& \sum_{l=0}^{n}\left(\begin{array}{c}
A_{m, m+2 l+1}^{(F)} \\
A_{m, m+2 l+1}^{(f)}
\end{array}\right) e_{1,61}^{(1)}+\left(+\begin{array}{c}
B_{m, m+2 l+1}^{(F)} \\
B_{m, m+2 l+1}^{(f)}
\end{array}\right) e_{1,62}^{(1)} \\
& \quad+\left(\begin{array}{c}
C_{m, m+2 l}^{(F)} \\
C_{m, m+2 l}^{(f)}
\end{array}\right) e_{1,63}^{(1)}+\left(+\begin{array}{c}
D_{m, m+2 l+1}^{(f)} \\
D_{m, m+2 l+1}^{(f)}
\end{array}\right) e_{1,64}^{(1)}=0 \quad(n=0,1,2, \ldots)
\end{aligned}
$$

where $e_{1, i j}^{(k)}$ are series expansion coefficients for the saturated porous media calculated in the same way as above. For the permeable boundary conditions at $r \leq a, 0 \leq \varphi \leq 2 \pi, \theta=\pi / 2$, for $m=0,1,2, \ldots$, $\sigma_{l, r r}^{(1)}=0$, we get

$$
\sum_{l=0}^{n}\left(+\begin{array}{c}
A_{m, m+2 l}^{(F)} \\
A_{m, m+2 l}^{(f)}
\end{array}\right) e_{l 1}^{(1)}+\left(+\begin{array}{c}
B_{m, m+2 l}^{(F)} \\
B_{m, m+2 l}^{(f)}
\end{array}\right) e_{l 2}^{(1)}=0 \quad(n=0,1,2, \ldots)
$$

where $e_{l, j}^{(k)}$ are series expansion coefficients when calculating the permeability of the saturated soil.

The above Eqs. $(39) \sim(46)$ constitute a system of homogeneous linear equations with the unknown coefficients $\left\{A_{m n}^{(1)}, B_{m n}^{(1)}, C_{m n}^{(1)}\right\},\left\{A_{m n}^{(2)}, B_{m n}^{(2)}, C_{m n}^{(2)}\right\}$ in the half-space and $\left\{A_{m n}^{(F)}, B_{m n}^{(F)}, C_{m n}^{(F)}, D_{m n}^{(F)}\right\}$ in the soft valley. Symbolically, the system of equations can be represented by

$$
\sum_{j=0}^{\infty} \mu_{i, j} x_{j}=n_{i} \quad(i=0,1,2, \ldots)
$$

where $\mu_{i j}$ represents the unknown coefficients of an infinite matrix , $x_{j}$ represents the unknown infinite sequence, and $n_{i}$ represents a known infinite sequence. Equations (47) are infinite for $i$ from 1 to $\infty$, and each equation for definite $i$ consists of the infinite sums. Therefore, the system of equations can be solved by means of truncating the infinite sums into finite sums. Then a finite matrix will be obtained. The number of terms considered should be large enough for satisfying the required accuracy.

\section{RESULTS AND DISCUSSION}

In this section, the effects of the wavelength and the angle of incidence of P-wave and the porosity of sediments in the hemispherical valley on the surface displacement response will be investigated. As the wave potentials are represented by the spherical coordinate as Eqs.(4), the displacements can be denoted by the spherical coordinate ${ }^{[15]}$. The amplitudes of the displacement vector $\left(u_{r}, u_{\theta}, u_{\varphi}\right)$ can be obtained from Eq.(14) and the potentials in Eqs.(19),(22) and (23). For the convenience of parametric study, a dimensionless frequency $\eta$ is introduced:

$$
\eta=\frac{2 a}{\lambda_{0}}=\frac{h_{0} a}{\pi}
$$

where $\lambda_{0}$ is the wavelength of the incident $\mathrm{P}$-wave. The properties of the saturated hemispherical alluvial valley and the elastic half-space are listed in Table 1 , where $A, N, R, Q$ are Biot parameters ${ }^{[12]}, \rho_{s}$ and $\rho_{f}$ are the mass densities of solid and liquid components, respectively, and $n$ is the porosity of soil, $\lambda$ and $\mu$ are Lame parameters of elastic material, and $\rho$ is the mass density of the half space.

Typical distributions of vertical surface displacement in the vicinity of the hemispherical alluvial valley induced by incident $\mathrm{P}$-wave at various angles of incidence and dimensionless frequencies are shown in Figs.3. In Figs.3, the $z$-axis represents the vertical displacement magnification, i.e. $M_{z}=u_{z} /\left|u^{i}\right|$, 
Table 1. The properties of the saturated hemispherical alluvial valley and the elastic half space

\begin{tabular}{|c|c|c|c|c|c|c|}
\hline \multicolumn{7}{|c|}{ saturated hemispherical alluvial valley } \\
\hline$A(\mathrm{~Pa})$ & $N(\mathrm{~Pa})$ & $R(\mathrm{~Pa})$ & $Q(\mathrm{~Pa})$ & $n$ & $\rho_{s}\left(\mathrm{kgm}^{-3}\right)$ & $\rho_{f}\left(\mathrm{kgm}^{-3}\right)$ \\
\hline $4.45 \times 10^{9}$ & $2.76 \times 10^{9}$ & $3.26 \times 10^{8}$ & $7.43 \times 10^{8}$ & 0.66 & $2.6 \times 10^{3}$ & $1.0 \times 10^{3}$ \\
\hline \multicolumn{7}{|c|}{ elastic half-space } \\
\hline & & $\lambda(\mathrm{Pa})$ & $\mu(\mathrm{Pa})$ & \multicolumn{3}{|c|}{$\rho\left(\mathrm{kgm}^{-3}\right)$} \\
\hline & & $22.4 \times 10^{9}$ & $29.9 \times 10^{9}$ & \multicolumn{2}{|c|}{$2.65 \times 10^{3}$} & \\
\hline
\end{tabular}

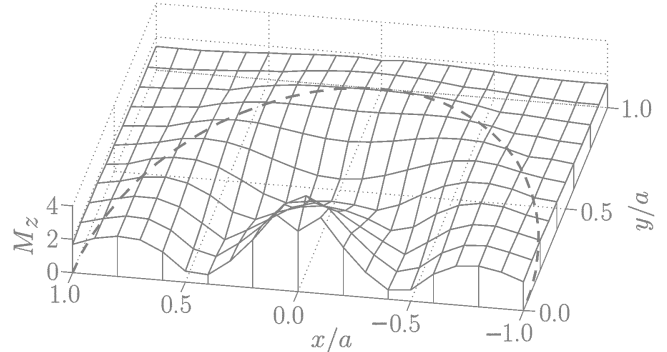

(a) $\gamma=0^{\circ}, \eta=0.5$

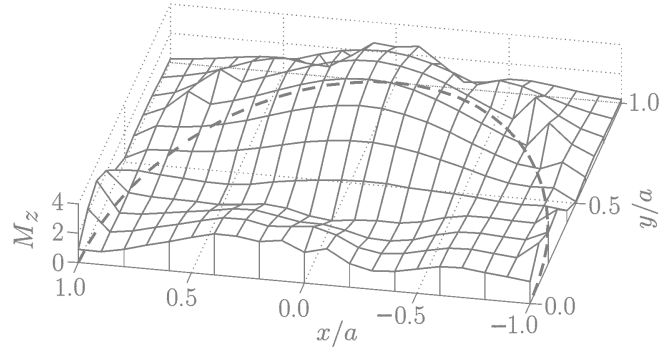

(c) $\gamma=30^{\circ}, \eta=0.5$

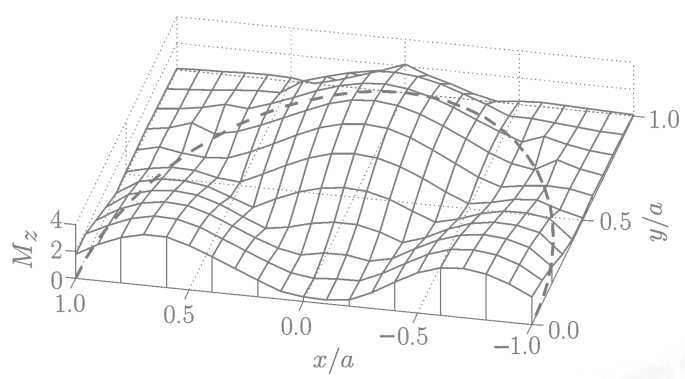

(e) $\gamma=85^{\circ}, \eta=0.5$

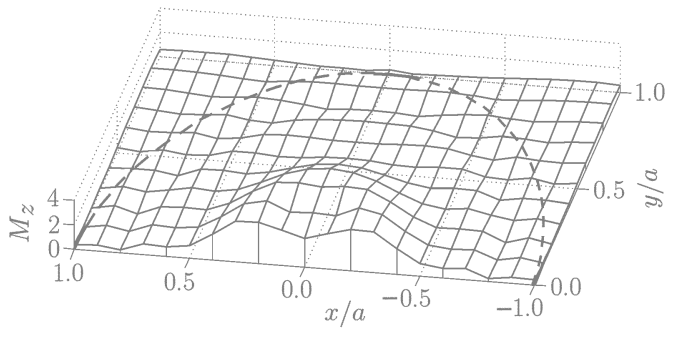

(b) $\gamma=0^{\circ}, \eta=1.5$

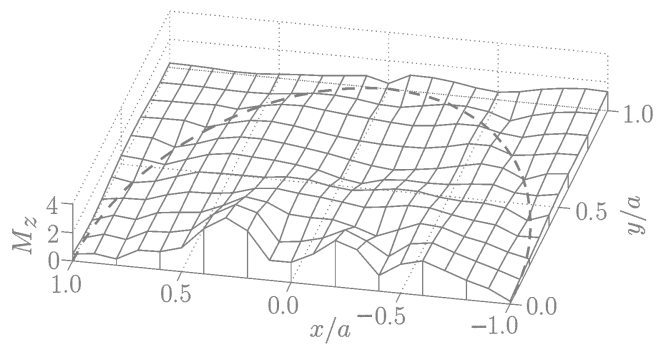

(d) $\gamma=30^{\circ}, \eta=1.5$

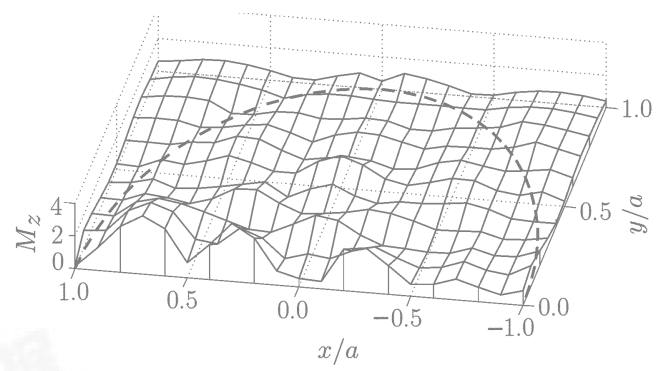

(f) $\gamma=85^{\circ}, \eta=1.5$

Fig. 3. The vertical displacement magnifications around the surface of saturated hemispherical alluvial valley induced by P-waves.

where $u_{z}$ is the vertical displacement of the surface induced by P-wave, and $\left|u^{i}\right|=a_{0}$ is the displacement amplitude of incident $\mathrm{P}$-wave. It can be seen from the figures that the displacement field becomes much more complicated for the presence of the hemispherical alluvial valley. For a fixed value of dimensionless frequency of incident wave, e.g. $\eta=0.5$ or 1.5 , with an increase of the angle of incidence, the influential zone of the maximum displacement magnifications transfers from the center to the edge of the hemispherical alluvial valley. The magnification effects at $\eta=1.5$ is become slightly smaller than those at $\eta=0.5$. Thus, the incidence angle and the dimensionless frequency of $\mathrm{P}$-wave have much influence on the pattern and amplitude of the surface displacements of the hemispherical alluvial valley. 
The distributions of horizontal displacement at the surface of the hemispherical alluvial valley induced by incident $\mathrm{P}$-wave with the incidence angle $\gamma=30^{\circ}$ and the dimensionless frequency $\eta=1.5$ are shown in Fig.4. Similar to the magnifications of the vertical displacement, the displacement magnifications in the $x$ - and $y$-directions can be expressed as $M_{x}\left(=u_{x} /\left|u^{i}\right|\right)$ and $M_{y}\left(=u_{y} /\left|u^{i}\right|\right)$ respectively, where $u_{x}$ and $u_{y}$ are the $\mathrm{P}$-wave induced surface displacements in the $x$ - and $y$-directions. Compared with the magnifications of vertical displacement (see Fig.3(d)), the displacements in $x$ - and $y$-directions are at an equal level of magnitude in the case of oblique incidence of $\mathrm{P}$-waves.

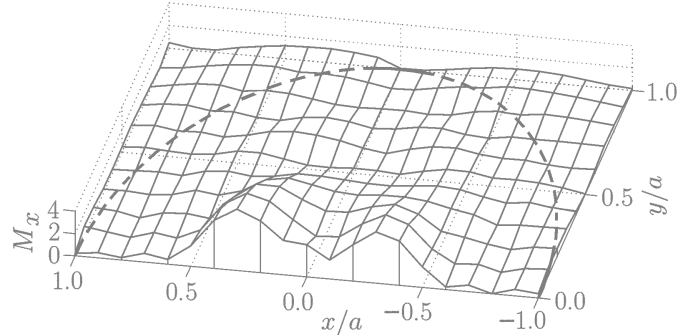

(a) $x$-component of displacement

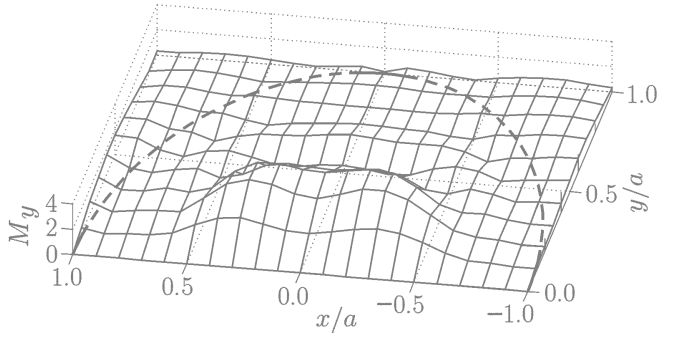

(b) $y$-component of displacement

Fig. 4. The magnification of $x$ - and $y$-component of displacements around the surface of saturated hemispherical alluvial valley for $\gamma=30^{\circ}$ and $\eta=1.5$.

It is also interesting to investigate the effects of the sediments porosity of the hemispherical valley on the surface displacement response. To this end, various values of the porosity of sediments in the hemispherical valley $n$ are chosen, i.e. $n=0.2,0.4,0.6,0.8$. The dimensionless frequency $\eta=1.5$ and the values of other parameters of the two media remain the same as listed in Table 1. Figure 5 shows the variation of the maximum values of vertical displacement magnifications $M_{z \max }$ with the porosity of sediments $n$ for normal incidence of $\mathrm{P}$-wave. It is indicated that the maximum magnifications of vertical displacements become higher with the increase of soil porosity.

Unlike Lee ${ }^{[9]}$, who stated that the displacement magnification at the surface of the alluvial valley, which was simulated by single-phase elastic theory, is not greater than that at the surface of elastic half-space (which is 2.0), the above investigations show that the displacement magnification effects will be much more obvious, especially for the sediment with high porosity, when the soil deposits in the hemispherical alluvial valley are simulated by Biot's dynamic theory. Therefore, it would be more reasonable to employ Biot's dynamic theory to simulate the saturated hemispherical alluvial valley when evaluating the $\mathrm{P}$-wave induced displacement responses.

\section{CONCLUSIONS}

Based on Biot's dynamic theory for saturated porous media, an analytical solution for threedimensional scattering and diffraction of plane P-wave by a hemispherical alluvial valley with saturated soil deposits is obtained by means of wave functions expansion technique. Based on this, the surface displacements around the soft alluvial valley are further analyzed. Numerical results show that the incidence angle and the dimensionless frequency of $\mathrm{P}$-wave have significent influence on the $\mathrm{P}$-wave induced displacement field, including its pattern and amplitude. The magnification effects of the surface displacement for higher dimensionless frequencies (e.g. $\eta=1.5)$ is slightly smaller than those at smaller ones (e.g. $\eta=0.5$ ). With the increase of the porosity of sediments, the displacement magnifications of the surface of alluvial valley become higher. When the hemispherical alluvial valley is assumed as a saturated porous medium, its displacement magnifications are much larger than those when it is 
assumed as a single-phase elastic medium. Therefore, for engineering practice, it is more reasonable to assume the alluvial valley a two-phase saturated porous medium, especially for the sediment with high porosity when analyzing the $\mathrm{P}$-wave induced displacement response of an alluvial valley.

\section{References}

[1] Singh,S.K.,Mena,E. and Castro,R., Some aspects of source characteristics of the 19 September 1985 Michoacan earthquake and ground motion amplification in and near Mexico City from strong motion data, Bulletin of Seismological Society of America, Vol.78, No.2, 1988, 451-477.

[2] Trifunac,M.D., Scattering of plane SH wave by a semi-cylindrical canyon, Soil Dynamics and Earthquake Engineering, Vol.1, 1973, 267-281.

[3] Todorovska,M. and Lee,V.W., Surface motion of shallow circular alluvial valleys for incident plane SH waves: analytical solution, Soil Dynamics and Earthquake Engineering, Vol.4, 1991, 192-200.

[4] Wong,H.L. and Trifunac,M.D., Surface motion of semi-elliptical alluvial valley for incident plane SH waves, Bulletin of Seismological Society of America, Vol.64, No.1, 1974, 1389-1408.

[5] Cao,H. and Lee,V.W., Scattering and diffraction of plane P waves by circular-cylindrical canyons with variable depth-to-width ratio, Soil Dynamics and Earthquake Engineering, Vol.9, No.3, 1990, 141-150.

[6] Lee,V.W. and Cao,H., Diffraction of SV by circular canyons of various depth, Journal of Engineering Mechanics, ASCE, Vol.115, No.9, 1989, 2035-2056.

[7] Mow,C.C. and Pao,Y.H., The diffraction of elastic waves and dynamics stress concentrations, Rand report, R-482-PR, New York, 1971.

[8] Lee,V.W., A note on the scattering of elastic plane waves by a hemispherical canyon, Soil Dynamics and Earthquake Engineering, Vol.1, No.3, 1982, 122-129.

[9] Lee,V.W. Three-dimensional diffraction of plane P, SV and SH waves by a hemispherical alluvial valley, Soil Dynamics and Earthquake Engineering, Vol.3, No.3, 1984, 133-144.

[10] Li,W.H. and Zhao,C.G., An analytical solution for the diffraction of plane P-waves by circular cylindrical canyons in a fluid-saturated porous media half space, Chinese Journal of Geophysics, Vol.46, No.4, 2003, 539-546 (in Chinese).

[11] Li,W.H. and Zhao,C.G., Scattering of plane P waves in alluvial valleys with saturated soil deposits, Chinese Journal of Geotechnical Engineering, Vol.25, No.3, 2003, 346-351 (in Chinese).

[12] Biot,M.A., Theory of propagation of elastic wave in fluid-saturated porous soil, Journal of the Acoustical Society of America, Vol.28, No.2, 1956, 168-178.

[13] Deresiewicz,H., and Rice,J.T., The effect of boundaries on wave propagation in a liquid filled porous solid.: Reflection of plane waves at a free plane boundary (non-dissipative case), Bulletin of Seismological Society of America, Vol.50, No.4, 1960, 599-607.

[14] Lee,V.W., Displacements near a three-dimensional hemispherical canyon subjected to incident plane waves, Report No.CE 78-16, Department of Civil Engineering, University of Southern California, Los Angeles, California, 1978.

[15] Dong,J. Study on the three-dimensional scattering and diffraction of plane-waves by irregular local sites with saturated soil, Master Dissertation (Supervisor: Zhao Chenggang), Beijing: Beijing Jiaotong Unversity, 2005 (in Chinese).

[16] Li,L. and Zhao,C.G., Equations of wave propagation with mass-coupling effect in fluid-saturated porous media, Acta Mechanica Solida Sinica, Vol.24, No.2, 2003, 243-248 (in Chinese). 